\title{
Enhanced FFR Scenario for Pilot Contamination Mitigation in 5G Systems with Massive Mimo \\ M.M.Badr, M.M.Fouda, A.S.T.El-dien
}

Electrical Engineering Dept., Faculty of Engineering, ShoubraUniv.,Egypt

E-Mail: mahmoud.m.ismail15@gmail.com

\begin{abstract}
Massive multiple-input multiple-output (MIMO) is the technology of the future, because it has the capability to increase a wireless system capacity without costing the system to purchase an additional bandwidth. It also has the capability to decrease the energy consumption of the system. All these capabilities have promoted the massive MIMO to be a promising technology for the fifth generation $(5 \mathrm{G})$ wireless communication systems. However, it has an important issue that limits the benefits which can be acquired by deploying it in 5G. This issue is the pilot contamination, which means the interference between pilot sequences. This occurs when the same pilot sequence is used by more than one terminal in the channel estimation phase at the same time and this causes interference between data symbols later. In this paper, we propose an enhanced fractional frequency reuse (FFR) scenario to mitigate the effects of pilot contamination by following an algorithm in assigning the pilot sequences to the system terminals instead of the random assignment. Simulation results show that our proposed scenario outperforms the traditional FFR in terms of signal to interference and noise ratio (SINR) and capacity.
\end{abstract}

Keywords: 5G, Massive mimo, Pilot contamination, Inter cell interference .

\section{Introduction}

The fifth generation $(5 \mathrm{G})$ is the next generation of wireless cellular networks that will support a larger capacity and a higher data rate than the previous generations through the emergence of new technologies and having a novel cellular architecture [1-3]. As the number of mobile subscribers and the wireless devices increases every year, and the size of multimedia contents such as images, audios and videos continuously increase, there is a continuous demand for a larger capacity wireless communication system that can support high data rates. In addition to that the world's trend is what is called green communication to eliminate the environment pollution and decrease the cost of electricity usage. The fourth generation (4G) system with its current technologies cannot face the future requirements, because the band dedicated for wireless cellular communication is nearly occupied and the $4 \mathrm{G}$ system was not built to be an energy efficient system. These aspects push the researchers to think beyond the $4 \mathrm{G}$ and to have a $5 \mathrm{G}$ system. Many researchers agree that massive multiple-input multiple-output (MIMO), cognitive radio, spatial multiplexing and millimeter wave communication are promising technologies to be used in the $5 \mathrm{G}$ system [1-3]. Massive MIMO technology is a very good technology to achieve the spectral and energy efficiencies requirements of the $5 \mathrm{G}$ system. MIMO is the technology of using multiple antennas at both the transmitter and the receiver. This has the effect of creating multiple communication channels from the same time-frequency channel, where each communication channel is identified by its propagation factor. MIMO technology is being used in the $4 \mathrm{G}$, but with limited equal numbers of antennas at both the base station (BS) and the mobile terminal for suitable terminal size. To maximize the benefits of MIMO, increase the system capacity and also keep small terminal size, massive MIMO technology has been proposed, in which the BS has a very large number of antennas to serve many single antenna terminals at the same time-frequency resources $[4,5]$. There are many challenges to be overcome for the practical deployment of massive MIMO such as pilot contamination, operation in frequency division duplex (FDD) mode, accurate propagation model and hardware impairments. The problem of pilot contamination [5-7] is considered as the bottleneck of the massive MIMO performance. This problem results during the process of channel estimation, if the same pilot sequence is used by more than one terminal at the same time. This problem causes interference during data transmission that remains existent even if we equipped the BS with an unlimited number of antennas. Due to its importance, the pilot contamination problem has been heavily investigated in many researches. In [810], a time shifted protocol has been proposed, that mitigates the problem by dividing the system into groups through transmitting the pilot sequences at non overlapping times from each group. This has the effect of decreasing the number of interfering cells by an order equals to the number of the system groups. In [11], precoding based method has been proposed that mitigates the problem through collaboration between the system BSs. In [12], fractional frequency reuse (FFR) method has been proposed that mitigates the problem through using different reuse factors for the same cell terminals. However, all these papers assign the available pilot sequences randomly to the terminals. In this paper, we propose an enhanced FFR scenario that takes the ability to acquire and track the large scale fading factors into account, while assigning the pilot sequences to the system terminals. Our proposed scenario enhances the minimum 
achievable per terminal capacity and in general, enhances the mean achievable capacity per terminal. The rest of the paper is organized as follows. In Sec. 2, we explain the problem of pilot contamination and the methodology followed in our proposed scenario to mitigate it. In Sec. 3, we present our simulation results and discuss them. In Sec. 4, we present our conclusions.

\section{Material and methods}

In this section, we model the problem of pilot contamination mathematically and explain the criteria followed by our proposed scenario to mitigate the effect of pilot contamination.

\subsection{Modeling the problem}

We built our system model inspired by the models of [5, 6] and [13]. We considered that our system composed of $L$ cells, each cell has a BS located in its center and equipped with $M$ antennas. The BS of each cell serves $K$ single antenna terminals that are uniformly distributed in the cell area, which is a hexagon of radius $\Gamma$. The propagation channel between any terminal (k) located in any cell (l) and the m-th antenna of the ith BS can be modeled as:

$$
g_{m i k l}=h_{m i k l} \sqrt{\beta_{i k l}},
$$

where it is a combination of small scale fading coefficient represented by $h_{\text {mikl }}$ and a large scale fading coefficient represented by $\sqrt{\beta_{i k l}}$, which is the same for all the $\mathrm{M}$ antennas at the i-th BS. $\beta_{i k l}$ remains constant for many coherence times and can be easily acquired and tracked according to $[11,14]$ and [15]. $\beta_{i k l}$ accounts for the shadow fading (SF) and the geometric attenuation and can be modeled as:

$$
\beta_{i k l}=\frac{z_{i k l}}{r_{i k l}{ }^{\gamma}}
$$

where $Z_{i k l}$ is a $\log$ normal random variable, which means that $\left(10 \log _{10} z_{i k l}\right)$ has a Gaussian distribution of zero mean and $\sigma_{S F}$ standard deviation), $r_{i k l}$ is the distance from the $\mathrm{k}$-th terminal of the 1-th cell to the i-th BS and $\gamma$ is the attenuation factor. Each BS must have the channel state information for all its terminals to be able to send/receive data to/from them. For this purpose, each BS must execute a channel estimation process. In our model, we followed the following algorithm:

1. The terminals of each cell send uplink orthogonal pilot sequences to its BS.

2. The BS process the received pilot sequences to estimate the propagation channels of its terminals.

3. By the assumption of channel reciprocity, the estimated channel can be used for both uplink and downlink.
Each terminal in the system must have an orthogonal (unique) pilot sequence for proper channel estimation process, but the number of orthogonal pilot sequences available each coherence time (the time within it, the channel is assumed unchanged) is limited. For a large capacity system, we have to reuse the same pilot sequences. In our model, we assumed that the same set of orthogonal pilot sequences $\left(\Phi=\left(\Psi \_1, \Psi \_2, \ldots, \Psi_{-} \mathrm{K}\right.\right.$ )) is reused among all the system cells and we also assumed simultaneous pilot transmission from all the system terminals (which is the worst case). Thus, each BS receives this set from all the system cells instead of its own cell only. This is called pilot contamination. The following equation models the received signal by the $\mathrm{i}$-th $\mathrm{BS}$ after pilot sequences transmission:

$$
Y_{i}=\sqrt{P_{p}} \sum_{l=1}^{L} G_{i l} \Phi^{T}+W_{i}
$$

where $P_{p}$ is the power used by each terminal in pilot transmission, $G_{i l}$ is the matrix of channel coefficients between the 1-th cell terminals and the i-th BS, $T$ means a matrix transpose and $W_{i}$ is the additive white noise. After channel estimation process at the $\mathrm{i}$-th $\mathrm{BS}$, the estimated channel matrix is not the actual one, but it is a linear combination of all channel matrices as shown in the following equation:

$$
\begin{aligned}
\overline{\mathrm{G}}_{i i}=\frac{1}{\sqrt{P_{p}} \tau} Y_{i l} \Phi^{*} & =G_{i i}+\sum_{l \neq i}^{L} G_{i l} \\
& +\frac{1}{\sqrt{P_{p}} \tau} W_{i} \Phi^{*}
\end{aligned}
$$

where $*$ means a matrix conjugate and $\tau$ is the length of each pilot sequence. After the channel estimation process, each BS is ready to exchange data with its terminal, but using this version of the estimated channel matrix by the BS in transmitting or receiving data causes inter-cell interference. After following a mathematical derivation as used in $[6,13]$, when the number of antennas at the BS tends to infinity, the estimated received data symbol by the i-th BS from its $\mathrm{k}$-th terminal is given by:

$$
\hat{x}_{k i}^{\wedge}=\sum_{l=1}^{L} \beta_{i k l} x_{k l}=\beta_{i k i} x_{k i}+\sum_{l \neq i}^{L} \beta_{i k l} x_{k l} \text {, }
$$

This estimated symbol is not the actual one, but it is a combination of the direct signal represented by the first term and inter-cell interference (the received data symbols from the $\mathrm{k}$-th terminals of all the other cells that used the same pilot sequence in the stage of pilot transmission) represented by the second term. The signal to interference and noise ratio (SINR) of the k-th terminal of the $\mathrm{i}$-th cell can be given by: 


$$
\operatorname{SINR}_{k}=\frac{\left(\beta_{i k i}\right)^{2}}{\sum_{l \neq i}^{L}\left(\beta_{i k l}\right)^{2}}
$$

This SINR can be translated to the capacity per terminal using Shannon relation:

$$
\begin{aligned}
& \mathrm{C} \_\mathrm{k}=(\mathrm{B} / \alpha)((\mathrm{T}-\tau-\mathrm{n}) / \mathrm{T})\left(\left(\mathrm{T} \_\mathrm{s}-\mathrm{T} \_\mathrm{c}\right) / \mathrm{T} \_\mathrm{c}\right) \log \_2(1+\llbracket \\
& \left.\mathrm{SINR} \rrbracket \_\mathrm{k}\right),
\end{aligned}
$$

where $B$ is the system bandwidth in megahertz, $\alpha$ is the frequency reuse factor, $\mathcal{T}$ is the coherence time expressed in terms of the number of symbols, $\tau$ is the number of symbols used for pilot transmission, $n$ is the number of symbols exhausted in signal processing, $T_{s}$ is the symbol duration in seconds and $T_{c}$ is the cyclic prefix in seconds.

\subsection{Proposing a scenario}

As we saw in Sec. 2.1, the problem of pilot contamination caused inter-cell interference that limits the capacity per terminal that can be achieved by the technology of massive MIMO. In this section, we show our proposed scenario to mitigate the effect of pilot contamination and obtain a higher capacity per terminal. Our proposed scenario combined the advantages of both the FFR scheme of [12] and the pilot allocation algorithm of [15]. In our scenario:

- All the system cells are connected to a network hub, which is responsible for assigning the pilot sequences to the system terminals.

- The terminals of each cell are categorized either center or edge terminals according to their distances from the cell BS.

- The set of pilot sequence is divided into four subsets: Center, Edge1, Edge2 and Edge 3 as shown in Fig (1).

- The Center is the subset used to assign pilot sequences to center terminals of all the cells with a reuse factor of one as shown in Fig (2).

- Edge1, Edge2 and Edge3 are the subsets used to assign pilot sequences to edge terminals of the cells with a reuse factor of three as shown in Fig (2) .

- Each BS estimates the large scale fading factors of all the system terminals and sends them to the network hub, which uses them as indicators for the propagation channels.

\begin{tabular}{|l|l|l|l|}
\multicolumn{1}{c}{ Center } & \multicolumn{1}{c}{ Edge1 } & \multicolumn{1}{c}{ Edge2 } & \multicolumn{1}{c|}{ Edge3 } \\
\hline & & & \\
\hline
\end{tabular}

Fig (1) The division of pilot sequences into four subsets.

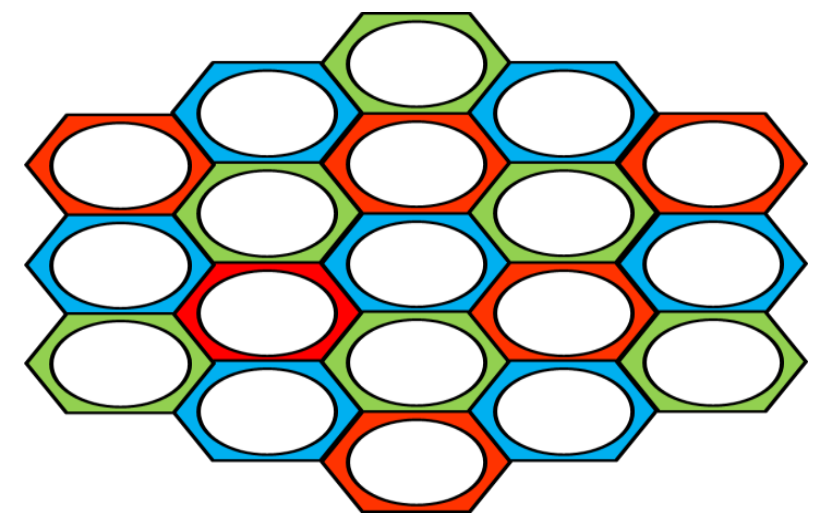

Fig (2) The division of each cell into center and edge areas.

For any $\left(\beta_{i k i}\right)^{2}$ :

- It is called the channel gain for the $\mathrm{k}$-th terminal, if $i=l$.

- It is called the level of interference caused by the $\mathrm{k}$-th terminal of the 1-th cell on the k-th terminal of the i-th cell, if $i \neq l$.

In the network hub, for a target cell i:

- The terminals of the $\mathrm{i}$-th cell are sorted according to their channel gain.

- The terminals of any other cell are sorted according to their interference level on the i-th cell.

- The network hub assigns the pilot sequences, so that the same pilot sequence assigned to a low channel gain terminal in the target cell is assigned to the other cells terminals of low interference respectively.

\section{Results and Discussion}

In this section, we evaluate the performance of our proposed scenario by presenting the results obtained from the computer simulations, which apply the criteria of our proposed scenario to mitigate the effect of pilot contamination. The simulation values assigned to our parameters are 
Table (1) Simulation Parameters.

\begin{tabular}{lc}
\hline parameter & value \\
\hline$L$ & 37 cells \\
$\Gamma$ & $1600 \mathrm{~m}$ \\
$B$ & $20 \mathrm{MHz}$ \\
$\gamma$ & 3.8 \\
$\sigma_{S F}$ & $8 \mathrm{~dB}$ \\
$\mathcal{T}$ & 7 OFDM symbols \\
$\tau$ & 2 OFDM symbols \\
$n$ & 1 OFDM symbol \\
$T_{S}$ & $71.4 \mu \mathrm{s}$ \\
$T_{c}$ & $4.76 \mu \mathrm{s}$ \\
\hline
\end{tabular}

\subsection{FFR}

When we modeled the problem of pilot contamination in Sec. 2.1, we used a reuse factor of one, which means that all the system cells interfere with each other. In his efforts to mitigate the problem of pilot contamination, Marzetta proposed to use a less aggressive frequency reuse factor of three to decrease the number of interfering cells [6]. This proposal enhances the performance of the low capacity terminals, but it eventually reduced the mean achievable per terminal capacity. FFR scheme was proposed in [12]. This scheme uses a reuse factor of three for the edge terminals, which are severely affected by the pilot contamination and a reuse factor of one to the center terminals. This scheme shows an improved performance compared to both a reuse factor of one and three. By applying our simulation parameters to the FFR scheme, we obtained the results shown in Fig. 3.

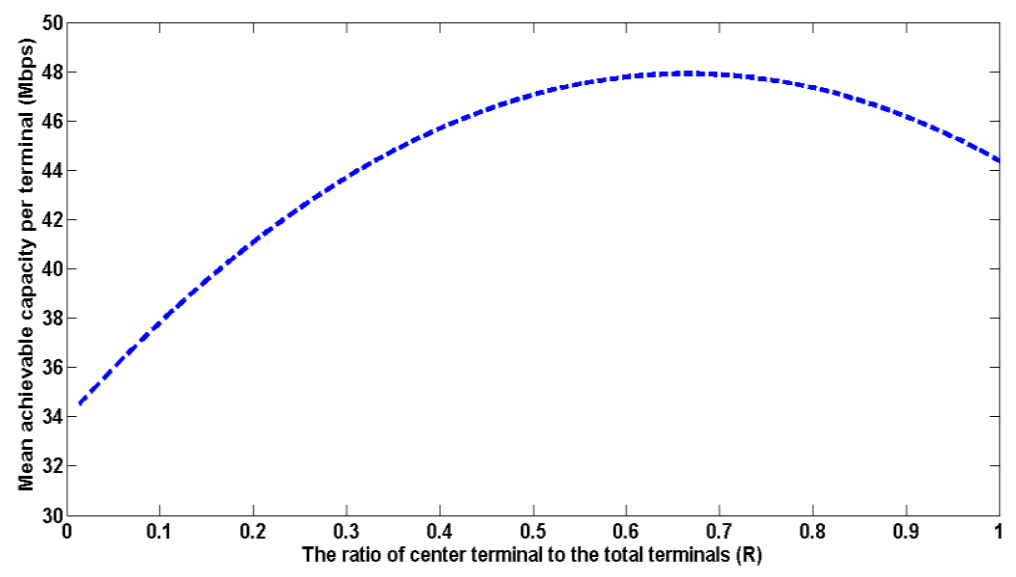

Fig (3) The mean achievable capacity per terminal at different values of R.

Fig (3) shows the mean achievable capacity per terminal at different values of $(\mathrm{R})$, which represents the ratio of center terminals to the total cell terminals. When $\mathrm{R}$ equals zero, this means that all the cell terminals are treated as edge terminals (i.e., the point on the curve corresponds to $\mathrm{R}=0$ represents the case of reuse factor of three). When $\mathrm{R}$ equals one, this means that all the cell terminals are treated as center terminals (i.e., the point on the curve corresponds to $\mathrm{R}=1$ represents the case of reuse factor of one). It is clear from Fig (3), that when the ratio $R$ is optimized at a certain value between zero and one, we obtain an FFR scheme that outperforms both a reuse factor of one and three.

\subsection{Enhanced FFR}

Instead of the random pilot sequences assignment to the system terminals followed by [12], our proposed scenario employs the pilot allocation algorithm of [15] to assign the pilot sequences to the system terminals. We should mention here that we do not adopt the scenario of [15] that uses a regular reuse factor of one, because we have already shown in Sec. 3.1 that the FFR is 
better than using regular reuse factors of one or three, but we only use their algorithm in assigning the pilot sequences to the terminals. By using the simulation parameters values given in Table 1 , we implemented our proposed scenario. In our simulation, we chose 10000 random positions for the terminals in each cell. For each terminal, we computed the SINR value and compared it with the one obtained if the traditional FFR scenario was used. We found that for nearly 8000 terminals out of the 10000 terminals, our proposed scenario gives higher performance than the traditional FFR of [12], but for the remaining 2000 terminals the FFR of [12] is better. We used the 10000 SINR values for each scenario to draw Fig (4).

Fig (4) compares the cumulative distribution function (CDF) of the SINR of our proposed scenario with the one of the FFR of [12]. In this fig, for any point in the range $(0,0.8)$ on the CDF axis, if we intersect the two curves and read the SINR values from the horizontal axis, we find that our proposed scenario provides higher SINR than the FFR of [12]. The two curves intersect at the point of 0.8 on the vertical CDF axis. This means that with probability of 20 percent (i.e., $1-0.8$ ) the FFR scenario will provide higher performance than our proposed scenario, which implies that our proposed scenario will provide higher performance than the FFR with probability of 80 percent. This can be interpreted that for each mobile terminal, our proposed scenario provides a higher SINR for nearly 80 percent of the time compared to the FFR of [12], but the FFR provides a higher SINR for only 20 percent of the time. In other words, we can say that our proposed scenario provides a higher SINR for nearly 80 percent of the target cell terminals versus the FFR that provides a higher SINR for only 20 percent of the target cell terminals. Thus, our proposed scenario enhances the performance of nearly 80 percent of the cell terminals and the other 20 percent of the terminals have a slightly lower performance compared to the FFR of [12]. This interprets the results given in Table 2 that shows that our proposed scenario enhances the mean achievable capacity per terminal compared to the FFR of [12]. It is clear also from Fig (4) that our proposed scenario improves the minimum achievable SINR and thus, it improves the minimum achievable capacity per terminal compared to the FFR of [12]. The enhancements provided by our proposed scenario and the abrupt increase of the curve of Fig (4) that represents our scenario are due to the following reasons:

- In our scenario, a low channel gain terminal in a target cell interferes only with the terminals of the other cells that cause low interference level and a high channel gain terminal in a target cell interferes only with the terminals of the other cells that cause high interference level. This makes the SINR values for most of the terminals convergent. This is not the case in[12].

- In the FFR of [12], a low channel gain terminal in a cell may interfere with the terminals of the other cells that cause high interference level, so a low channel gain terminal may suffer from a very low SINR and vice versa. This makes the SINR values are not convergent as in our scenario.

Table (2) Comparison of the mean per terminal capacity of the FFR of [12] and that of our proposed scenario.

\begin{tabular}{ccc}
\hline Scenario & FFR & Proposed \\
\hline Mean capacity per terminal & $46 \mathrm{MHz}$ & $75 \mathrm{MHz}$ \\
\hline
\end{tabular}

\section{Conclusions}

We have introduced the problem of pilot contamination being a substantial challenge that hampers the deployment of massive MIMO technology in the $5 \mathrm{G}$ wireless communication systems with its full powers. We presented our proposed scenario to mitigate the effects of pilot contamination. Our proposed scenario enhanced the performance of the traditional FFR by assigning the pilot sequences to the terminals, so that the terminals that suffer from low channel gain in a certain cell do not interfere with the other cells terminals that cause high interference level. Using computer simulation, we compared our proposed scenario with the traditional FFR and the results showed the ability of our proposed scheme in enhancing both the minimum achievable capacity per terminal and the mean achievable capacity per terminal. 


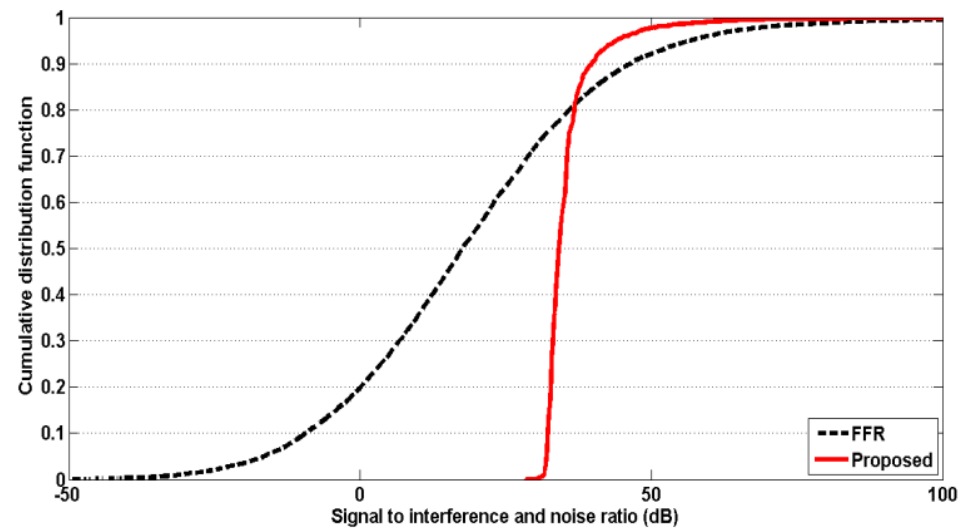

Fig (4) The cumulative distribution function of the SINR per terminal for both the FFR scheme of [12] and our proposed scenario

\section{References}

[1] C.-X.Wang et al., "Cellular architecture and key technologies for $5 \mathrm{G}$ wireless communication networks," IEEE Communications Magazine, vol. 52( 2), pp. 122,2014.

[2] J.G.Andrews et al., "What will 5G be?," IEEE Journal on Selected Areas in Communications, vol. 32 ( 6) , pp. 1065, 2014.

[3] F.Boccardi, R.W.Heath, A.Lozano, T.L.Marzetta, and P.Popovski, "Five disruptive technology directions for 5G," IEEE Communications Magazine, vol. 52, (2), pp. 74, 2014.

[4] E.G.Larsson, O.Edfors, F. Tufvesson, and T. L. Marzetta,"Massive MIMO for next generation wireless systems," IEEE Communications Magazine, Vol. 52(2), pp. 186, 2014.

[5] L.Lu, G.Y.Li, A.L.Swindlehurst, A.Ashikhmin, and R. Zhang, "An overview of massive MIMO: Benefits and challenges," IEEE Journal of Selected Topics in Signal Processing, vol. 8(5), pp. 742, 2014.

[6] T.L.Marzetta, "Noncooperative cellular wireless with unlimited numbers of base station antennas," IEEE Transactions on Wireless Communications, vol. 9(11), pp. 3590, 2010.

[7] K.Zheng et al.,"Survey of large-scale MIMO systems," IEEE Communications Surveys and Tutorials, vol. 17(3) , pp. 1738, 2015.

[8] K.Appaiah, A.Ashikhmin, and T.L. Marzetta, Pilot contamination reduction in multi-user TDD systems," in Proc. IEEE International Conference on Communications (ICC), 2010.

[9] F.Fernandes, A.Ashikhmin, and T.L. Marzetta, "Interference reduction on cellular networks with large antenna arrays," in Proc. IEEE International Conference on Communications (ICC), 2012.

[10] F.Fernandes, A.Ashikhmin, and T.L. Marzetta,"Inter-cell interference in noncooperative TDD large scale antenna systems," IEEE Journal on Selected Areas in Communications, vol. 31(2), pp. 192, 2013.

[11] A.Ashikhmin and T.Marzetta, "Pilot contamination precoding in multi-cell large scale antenna systems," in Proc. IEEE International Symposium on Information Theory (ISIT), 2012.

[12] X.Yan, H.Yin, M.Xia, and G.Wei, "Sequences Allocation in TDD Massive MIMO Systems," in Proc. IEEE Wireless Communications and Networking Conference (WCNC), 2015.

[13] M.M.Badr, M.M.Fouda, and A.S.Tag Eldien,"A novel vision to mitigate pilot contamination in massive MIMO-based 5G networks," in IEEE Proc. International Conference on Computer Engineering and Systems (ICCES), 2016.

[14] X.Zhu, Z.Wang, L.Dai, and C.Qian, 2015, "Smart pilot assignment for massive MIMO," IEEE Communications Letters, vol. 19, (9), pp. 1644.

[15] M. Alkhaled, E.Alsusa, and K. A.Hamdi, “A new allocation algorithm for pilot contamination mitigation in TDD massive MIMO systems," in Proc. IEEE Wireless Communications and Networking Conference (WCNC, 2017. 